\title{
SEVERE MALARIA CAUSED BY PLASMODIUM VIVAX IN PREGNANCY MASQUERADING AS HELLP SYNDROME
}

\author{
Monika Trivedi ${ }^{1}$, Premila Taneja ${ }^{2}$, Geetika Trivedi ${ }^{3}$, Pranjal Pankaj ${ }^{4}$
}

\section{HOW TO CITE THIS ARTICLE:}

Monika Trivedi, Premila Taneja, Geetika Trivedi, Pranjal Pankaj. "Severe Malaria caused by Plasmodium Vivax in Pregnancy Masquerading as HELLP Syndrome". Journal of Evolution of Medical and Dental Sciences 2014; Vol. 3, Issue 02, January 13; Page: 526-529, DOI:10.14260/jemds/2014/1860

\begin{abstract}
The following is a case report of a woman who presented to the hospital with severe anemia with features of HELLP syndrome and initial slide for P.vivax negative but on constant observation and monitoring turned out to be a case of severe malaria caused by vivax species of malaria. Primigravida with 35week5day pregnancy was admitted with complaints of severe anemia and history of fever 14 days back. Initially 3 pint blood was transfused i/v/o severe anemia. Investigations revealed $\mathrm{Hb}-3 \mathrm{gm} \%$ and platelet count 30, 000, LFT deranged, Hemolysis in peripheral smear, smear negative for malarial parasite. HELLP syndrome was in mind and this required termination but on repeat investigations and smear vivax was positive. Her platelet count kept deteriorating despite transfusions till antimalarial regimen was not started. Once smear positive the fastest antimalarial artesunate regimen with clindamycin was started and after $48 \mathrm{hrs}$. her counts improved. Overall 14 pints of platelets were transfused and patient delivered uneventfully with mild PPH at platelet count of 30,000 . Severe malaria is usually caused by $\mathrm{p}$ falciparum but recently reports of severe malaria caused by vivax is increasing and HELLP syndrome is an important differential diagnosis which needs to be excluded as management is entirely different.
\end{abstract}

KEY WORDS: Malaria, Plasmodium vivax, HELLP syndrome, Preeclampsia, Thrombocytopenia.

INTRODUCTION: Malaria in pregnancy is frequently underestimated as a public health problem by clinicians and public at large. It is one of the major causes of maternal and fetal morbidity and mortality worldwide ${ }^{1}$. P.vivax causing severe malaria is rare and only few cases are reported ${ }^{1}$. HELLP syndrome is an important $d / d$ of complicated malaria which needs to be excluded in view of completely different management.

CASE REPORT: A Primigravida with 35 week's 5 days period of gestation was admitted with complaints of mild pain in Abdomen, dyspnea, fatigue, breathlessness, and swelling all over the body. She had no previous antenatal checkups but Tetanus Immunization had been done. No other high risk factor was present in her history. She revealed history of high grade fever which started 14 days back with chills and rigor but no fever for past 3-4 days. No complaint of headache, epigastric pain or blurring of vision. She had widal positive private laboratory report.

On examination-GC-Poor, Anasarca present, markedly pale, clinically around $3 \mathrm{gm}$. \%, Tachycardia, BP-130/84 mm of Hg, Apyrexial, RR-28/min, chest-clear, CVS-Flow murmur present.

Per Abdomen Examination-uterus 30 weeks, longitudinal lie, cephalic presentation, FHS136/min/regular, liquor adequate, relaxed.

Per/Speculum Examination-NR, Per/Vaginal Examination-Multiparous os. 
Patient was admitted, kept propped up, oxygen was given by mask, Injection ceftriaxone 1 gm. IV BD, 3 pint RCC transfused under Lasix cover, Investigations sent in emergency. Blood Pressure monitoring, TPR Charting, Input /output monitoring was done. Investigations showed $\mathrm{Hb}$ 3gm\%, Platelet count-30, 000, TLC-23, 000, Liver Function Test -SGOT-67, SGPT-75(deranged), Kidney Function Test -Normal, Blood Urea -WNL, Serum electrolytes-WNL, peripheral smearhemolytic smear with fragmented \& target cells. Urine protein was in traces. Peripheral Blood Smear for Malarial parasite-Negative, WIDAL-Negative.

Day 2 - BP -110/70-130/84 mm of Hg (24 hrs. observation). Her pallor and anasarca improved, her $\mathrm{Hb}-7 \mathrm{gm} \%$ but Platelet count dropped to 25, 000 within 24 hours. HELLP Syndrome was suspected (Hemolysis, Elevated liver enzymes and low platelet count) which requires urgent termination. Normal BP was going against preeclampsia. Steroid coverage was given for fetal lung maturity in case urgent termination is required. 4 pint of platelets transfused. $\mathrm{Hb}-7 \mathrm{gm} \%$ but Platelet count dropped to 25,000 .

On Day 3 her LFT were deranged, and platelets dropped to 15, 000.0n peripheral blood smear plasmodium vivax came positive and patient was overall symptomatically better with no petechiae, rashes or bleeding complaint. She had two to three episode of fever (100 Degree F).

Immediately Artesunate regimen as per protocol(1.2gm iv stat followed by repeat dose at $12 \mathrm{hrs}$ then $24 \mathrm{hrly}$ for 5 days) was started for severe malaria with clindamycin $600 \mathrm{mg}$ iv TDS, 4 pint of platelets where transfused urgently, decision for termination withheld and fetal monitoring done (CTG\& BPS with Doppler normal with no evidence of retroplacental hemorrhage).

Day4, Patient had labor pains and P/V- cervical os 3-4cm dilated, 70\% effaced and vertex at 0 station. Repeat platelet count $-20,000$ post 8 pint platelets transfusions. No active intervention done to augment labor.

10 pint platelets arranged and transfused and high risk prognostication done, spontaneous delivery awaited with due precaution for active management of 3rd stage and prophylaxis for PPH. Intermittent fetal monitoring done with CTG. Patient delivered on Day-4 with mild PPH, platelets at delivery 30, 000 and then $48 \mathrm{hrs}$ post malarial regimen Platelet count started increasing. Apgar normal, EFW-2.2kg.

On discharge $-\mathrm{Hb}-9.7 \mathrm{gm} \%$, Platelet count-75, 000

DISCUSSION: About 1.5 million confirmed cases of Malaria are reported annually by national vector borne diseases control program of which $40-50 \%$ due to P.Falciparum. Women with malaria should have the severity of their condition assessed and documented as an aid to management ${ }^{2}$. Severity determines treatment and helps predict fatality rate. In uncomplicated Malaria fatality rates are low (0.1\% for P.Falciparum).In severe malaria, particularly in pregnancy fatality rates are higher (15$20 \%$ in non-pregnant as compared to $50 \%$ in pregnancy) ${ }^{3,4}$.Severe malaria include impaired consciousness, Convulsions, renal failure s.creatinine $>3 \mathrm{gm} / \mathrm{dl}$ ), jaundice (s.bil $>3 \mathrm{mg} / \mathrm{dl}$ ), severe anemia $(\mathrm{Hb}<5 \mathrm{gm} / \mathrm{dl})$, pulmonary edema, hypoglycemia, metabolic acidosis, shock, DIC mainly attributed to P.falciparum. P.vivax causing severe malaria is rare and only few cases are reported ${ }^{4}$. ].In one study about $68 \%$ of patients with vivax malaria had thrombocytopenia of which $32 \%$ had moderate to severe thrombocytopenia ${ }^{5}$.

Urgent microscopy, Rapid diagnostic tests are required. Microscopy is the gold standard but it could be negative in pregnancy known as "placental malaria". In some areas the majority of 
pregnant women with clinically significant malaria have a negative malaria blood film this is in contrast to nonpregnant adults where a competently read negative slide virtually rules out the diagnosis. It is possible that new antigen based RDT will prove more sensitive than conventional microscopy in detecting placental malaria but with limitations. Placental malaria can lead to profound anemia with few other symptoms and few clues in the lab tests. An afebrile woman with no parasite seen on a peripheral blood film may have placental malaria either as a contributing factor or sole cause of anemia ${ }^{1}$.

Severe malaria in pregnancy should be treated as non-pregnant ${ }^{1}$.Treatment of severe malaria is an emergency and required to get antimalarial drug levels as soon as possible, ideally by parenteral treatment (iv quinine or iv artesunate)or i/m quinine or artemether ${ }^{2,3}$.No trials were done in pregnant women that can demonstrate whether quinine or artemisine are preferable. However, in non-pregnant adults studies suggest that they are of comparable efficacy ${ }^{3}$. Advantage of artimisinin therapy is, it's the fastest acting antimalarial, leads to more rapid clearance of parasites and more effective in quinine resistant areas. Artesunate kills circulating ring stage parasite and so prevents sequestration which quinine does not. WHO recommends IV artesunate as first line drug3.Pregnant women are at increased risk of hypoglycemia with severe malaria and this should be looked for actively whenever a women deteriorates or shows behavioral changes.

Incidence of congenital malaria is 8-33\%.Infection of newborn can occur despite appropriate treatment in mother during pregnancy. All neonates whose mothers developed malaria in pregnancy should be screened for malaria with standard microscopy of thick and thin blood films at birth and weekly blood films for 28 days. It is useful to allow early detection and treatment of congenital malaria ${ }^{3}$.

Malaria may be complicated by development of thrombocytopenia, elevated liver enzymes, and/or hemolysis, which may be difficult to distinguish from HELLP (hemolytic anemia; elevated liver enzymes; low platelet count) syndrome in a pregnant patient 6,7 .HELLP syndrome is present in $20 \%$ cases of preeclampsia and requires urgent termination. It is an important differential diagnosis for severe malaria 7 .Careful observation is required for accurate diagnosis as HELLP syndrome requires urgent termination while Malaria with pregnancy does not need termination for maternal indication.

Hence vivax malaria initially thought benign can result in severe disease with increasing incidence (68\%) and moderate -severe thrombocytopenia in 32\%.HELLP syndrome is an important differential diagnosis and needs to be excluded.

\section{REFERENCES:}

1. Singh N, Shukla MM, Sharma VP. Epidemiology of malaria in pregnancy in central India. Bull World Health Organ 1999; 77:567-72.

2. National vector borne disease control programme; www.nvbdcp.gov.in/.

3. World Health Organization, authors. Guidelines for the Treatment of Malaria. Geneva: World Health Organization; 2006.

4. Nicholas Day and Arjen M. Dondorp. The Management of Patients with Severe Malaria Defining and Defeating the Intolerable Burden of Malaria III: Progress and Perspectives: Supplement to Volume 77(6) of American Journal of Tropical Medicine and Hygiene. 
5. Pranjal Pankaj, K. Chandran, Shrawan Kumar, Manish Kumar Singh. "Occurrence of thrombocytopenia in vivax malaria". Journal of Evolution of Medical and Dental Sciences 2013; Vol2, Issue 34, August 26; Page: 6458-6465

6. Ducarme G, Thuillier C et al; Malaria in Pregnant woman masquerading as HELLP syndrome; Am J Perinatol: Feb 2010:27(2):171-2.

7. Castillo Medina NM, Velazquez Fonseca et al; Gestational Malaria: HELLP syndrome Mistaken diagnosis; Ginecolobstet Mex; May 2008:76(5):287-91.

\section{AUTHORS:}

1. Monika Trivedi

2. Premila Taneja

3. Geetika Trivedi

4. Pranjal Pankaj

\section{PARTICULARS OF CONTRIBUTORS:}

1. Assistant Professor, Department of Obstetrics and Gynaecology, Rama Medical College Hospital and Research Centre, Kanpur.

2. Professor, Department of Obstetrics and Gynaecology, Rama Medical College Hospital and Research Centre, Kanpur.

3. Consultant, Department of Obstetrics and Gynaecology, Max Hospital, Delhi.
4. Assistant Professor, Department of Medicine, Rama Medical College Hospital and Research Centre, Kanpur.

\section{NAME ADDRESS EMAIL ID OF THE} CORRESPONDING AUTHOR:

Dr. Monika Trivedi, Assistant Professor, Department of Obstetrics and Gynaecology, Rama Medical College and Hospital,Kanpur. Add - 128/2/127, Yashoda Nagar, Kanpur - 208011.

Email - drmonikatrivedi@gmail.com

Date of Submission: 05/12/2013. Date of Peer Review: 06/12/2013.

Date of Acceptance: 30/12/2013. Date of Publishing: 13/01/2014. 